\title{
Changes in regional cerebral blood flow
}

\section{due to cognitive activation among patients}

\section{with schizophrenia}

\author{
B. K. TOONE, C. I. OKOCHA, K. SIVAKUMAR and G. M. SYED
}

\begin{abstract}
Background The regional cerebral blood flow $(\mathrm{rCBF})$ response to the Wisconsin Card Sort Test (WCST) has been used to assess the functional integrity of the prefrontal cortex in patients with schizophrenia.
\end{abstract}

\begin{abstract}
Aims In this study, patients were divided into two groups according to whether they had made few or many perseverative errors on a modified version of the WCST. A control group consisted of normal volunteers. The groups were then compared with respect to $\mathrm{rCBF}$ response to WCSTactivation.
\end{abstract}

\section{Method rCBF was measured during administration of a modified version of the WCSTand during a card sorting control task, using single photon emission computerised tomography (SPECT).}

\section{Results Performance of the modified WCST was associated with a widespread and substantial increase in $\mathrm{rCBF}$, particularly in the frontal region. The poorly performing group of patients with schizophrenia showed only a modest increase in $\mathrm{rCBF}$ in the left anterior cingulate region.}

\section{Conclusion Subjects with schizophrenia are able to respond to specific neuropsychological challenge with activation of the frontal regions.}

\footnotetext{
Declaration of interest C.I.O. was funded by the South East Thames Regional Health Authority Locally Organised Research Scheme.
}

The possibility that frontal lobe mechanisms may be dysfunctional in schizophrenia has been investigated using neuropsychological (Wisconsin Card Sorting Test, WCST; Berg, 1948) and functional neuroimaging (regional cerebral blood flow, rCBF) techniques. Weinberger et al (1986) reported a failure of prefrontal rCBF activation with WCST in a group of chronic unmedicated sufferers from schizophrenia with normal resting rCBF. However, the extent to which these findings can be generalised, and the relationship between $\mathrm{rCBF}$ and WCST performance, remain uncertain.

In attempting to address these questions, we identified two groups of subjects with schizophrenia: one performing adequately, and one poorly, on the WCST. Normal volunteers constituted a third group. An indirect measure of rCBF was obtained using a radiotracer uptake ratio. The radiotracer 99m-technetium-labelled hexamethylpropylene-amineoxime $\left({ }^{99 \mathrm{~m}} \mathrm{Tc}-\mathrm{HMPAO}\right)$ is used in a multidetector single photon emission computerised tomography (SPECT) scanning system during performance of the WCST and during a motor control task. The change in rCBF between the two occasions was attributed to the effects of cognitive activation.

\section{METHOD}

\section{Subjects}

\section{Screening procedure}

All male patients who were attending psychiatric out-patient clinics at Maidstone Hospital and at King's College Hospital, London and who met initial inclusion criteria were screened. Maidstone Hospital provides a service for a medium-sized county town and surrounding rural area; King's College Hospital lies in an inner-city area. Each clinic is part of a local community service. The initial inclusion criteria were as follows: (a) a diagnosis of schizophrenia according to DSM-III-R criteria (American Psychiatric Association, 1987);

(b) age 18-55;

(c) right-handedness (assessed using the Annett Handedness Schedule; Annett, 1970);

(d) no history of neurological disorder, hypertension (diastolic $90 \mathrm{mmHg}$ ), drug or alcohol abuse according to CAGE criteria (Mayfield et al, 1974), significant physical illness, drug treatment other than antipsychotic and anticholinergic medication, or psychiatric illness other than schizophrenia; and

(e) IQ above 75 as measured by the National Adult Reading Test (NART; Nelson, 1982) and Schonell graded word reading test (Schonell, 1942).

Eighty-four subjects met the above criteria and were invited to participate in the study: 72 accepted. Each subject completed Raven's Progressive Matrices (RPM; Raven, 1988), the Nelson modification of the WCST (Nelson, 1976), the controlled oral word association test (FAS), and the SET test (Isaacs \& Akhtar, 1972). The negative symptom rating scale (NSRS; Iager et al, 1985) and the Manchester scale (MS; Krawiecka et al, 1977) were employed as measures of clinical morbidity.

We wished to generate two groups of patients, each of which would perform adequately according to a measure of general intelligence, but one of which would perform adequately and the other poorly on the modified WCST.

The RPM was selected as a widely used measure of general intelligence that was not overly dependent on frontal mediation. Members of either patient group had to have a score exceeding the 50th percentile.

The WCST generates three sets of scores: the number of categories attained (CA), the number of perseverative errors (PE), and the total number of errors (TE). The perseverative error score has often been taken as the more specific measure of frontal lobe dysfunction, and a cut-off score of $4 / 5$ was chosen, so that those patients scoring 5 and above formed the poorly performing schizophrenia (PPS) group and those scoring 4 and below formed the adequately performing schizophrenia (APS) group. All our patients were able to complete the test.

The normal control group was recruited from healthy staff volunteers. 
Apart from not having a diagnosis of schizophrenia, they fulfilled the same inclusion criteria as the patient groups, and were asked to perform the same cognitive tests. Only subjects who obtained a score on the RMP exceeding the 50th percentile were considered.

All subjects were assessed by the same investigator (C.I.O.)

\section{Group characteristics}

Twelve patients with schizophrenia met the criteria for the APS group and $\mathbf{1 1}$ for the PPS group: the remainder failed to obtain an adequate score on the RPM. Eleven healthy volunteers formed the control group. The mean ages for the APS and PPS groups were 33.7 (s.d.=4.8) and 38.1 years $($ s.d. $=10.0)$ respectively. The mean age of the control group was 29.9 years (s.d.=6.2).

All 23 patients were receiving 'typical' antipsychotic drugs, and three were also receiving anticholinergic drugs. No other drugs were prescribed. The antipsychotic drug dose at the time of neuroimaging was recorded and converted into chlorpromazine equivalents. Age and antipsychotic medication were each entered as covariates in the statistical analysis. The mean age at onset of the illness (first medical contact) was 23.7 years, and the mean duration, 9.9 years for the APS group and 25.8 and 12.3 years for the PPS group. Members of the APS group had an average of 2.9 admissions and the PPS 4.0 admissions. These differences were insignificant.

\section{The cognitive task}

Weinberger et al (1986) used the standard form of the WCST. In a small pilot group of subjects with schizophrenia (none of whom took part in the subsequent study) we compared the standard and the modified forms. We found that subjects scored similarly on both, but were better able to tolerate the modified procedure, so we therefore adopted it for the main study. The WCST was performed twice by those subjects who entered the neuroimaging phase of the study: initially during screening, and again during imaging. In most instances several months elapsed between tests.

\section{Image acquisition \\ ${ }^{99 \mathrm{~m}} \mathrm{Tc}$-HMPAO, when given intravenously, crosses the blood-brain barrier at first pass:}

$4-5 \%$ of the injected dose enters the cerebral parenchyma and is temporarily trapped, due to a structural and conformational change that reduces its lipophilicity. The topographical distribution of HMPAO thus reflects rCBF during the 4minute period that immediately follows injection. Due to slow wash-out time, this amount remains fairly constant for several hours after injection (Sharp et al, 1986).

While the isotope was prepared, a heparinised cannula was introduced into the left antecubital vein. The instructions were read aloud by the investigator. The subject sat at a table. The test room was sound-proofed and air-conditioned. In the WCST the subject held the pack of cards in the left hand and sorted with the right hand. During the control task, the subject held the pack in the left hand and randomly distributed the cards to form four piles. The subject used the same number of cards and was encouraged to distribute them at approximately the same pace as during the card sort. An injection of $250 \mathrm{MBq}$ of the radiotracer was given 5 minutes after the card sort had commenced. The subject was not screened from the injection and was aware of the timing of the injection. Challenge and control procedures were administered in counterbalanced order on separate days.

Regional cerebral blood flow was measured using a multidetector head-dedicated tomography system, model SME 810 (Strichman Medical Equipment, MA, USA). The SME 810 has a resolution of about $10 \mathrm{~mm}$ full width at half maximum and an in-plane resolution of $14 \mathrm{~mm}$. Total count per slice was approximately 1000000 ; typical acquisition time was 15 minutes per slice. Reconstruction was carried out using the inverse countdependent Wiener high-resolution filters and the iterative method with the software version SME 2.66 (Strichman Medical Equipment).

\section{Image analysis}

${ }^{99 m}$ Tc-HMPAO SPECT studies can only provide relative $\mathrm{rCBF}$ data in terms of ratios because not enough is known about the kinetics of the tracer to enable absolute quantitation. Semi-quantitation in relative terms is done by 'normalising' the regional deposition or uptake of tracer (i.e., count density in a region of interest, ROI) to the deposition or uptake in a reference region. The choice of reference region is often based on the assumption that $\mathrm{rCBF}$ in that region is normal and that the region is not involved in the pathological process (Holman, 1986; Syed et al, 1992). In this study we chose the cerebellum as the reference region. The analyses were carried out by the same rater (C.I.O.), who was unaware of the group identity of the subject. Seven to eight overlapping slices (slice thickness $7.7 \mathrm{~mm}$ ) of the brain at, and parallel to, the orbitomeatal line were acquired. Three slices were chosen for the quantification of relative rCBF. These slices and the ROIs were identified on the transverse SPECT images with the help of a computed tomographic atlas, as described by Montaldi et al (1990). The slices were: (a) a transverse slice at the level of the orbitomeatal line, to demonstrate the cerebellum; on this slice a large ROI was delineated over the whole cerebellum; (b) a midventricular slice at $50 \mathrm{~mm}$ and parallel to the orbitomeatal line (Fig. 1); to improve the reproducibility of the method, a template was devised so that when fitted onto the slice it divided the whole slice into three in the frontal-occipital dimension; (c) a supraventricular slice $70 \mathrm{~mm}$ above the orbitomeatal line (Fig. 1), which was divided into four quadrants, using a template.

The regions thus delineated on each of these slices are as follows. The predominantly cortical areas were identified as a peripheral rim 5 pixels $(7.7 \mathrm{~mm})$ in width. In the midventricular slice, a frontal pole cortical segment 3 pixels in width was also identified. This corresponds approximately to the Brodmann area 10 . The cortical regions in the midventricular slice thus delineated were (in medial, anterior and posterior order): frontal pole,

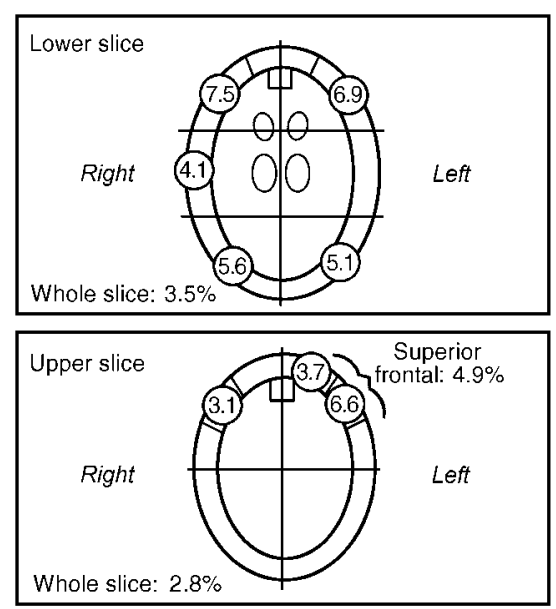

Fig. I Percentage increases in regional cerebral blood flow following cognitive activation. 
inferior prefrontal, superior temporal, and inferior parietal-occipital cortices. Additional irregular regions were delineated to incorporate two subcortical structures, the caudate and thalamus, on each side.

The supraventricular slice was selected to display the superior frontal and parietal cortices. The slice was divided into four equal quadrants. The cortical areas were defined as in the lower slice. The frontal cortical area was further subdivided into three equal segments. The two more anterior subsegments corresponded to the superior prefrontal cortex, the third segment to the superior motor and premotor cortices. The posterior segment corresponded to the superior parietal cortex. A rectangular ROI 5 pixels long (anterior-posterior) and 3 pixels wide was placed over areas of increased activity on the mesial surface of the frontal lobe, corresponding to the anterior cingulate region.

\section{RESULTS}

\section{Cognitive differences between groups: three group analyses}

Group selection was determined by the performance on the RPM and by the number of perseverative errors on the WCST. Accordingly, the groups did not differ on the former, while the PPS group performed poorly on the latter (Table 1). The PPS group also attained fewer WCST concepts and a higher total error score than the other groups. Neither group of patients with schizophrenia performed as well as the normal control group on the NART, FAS, or SET.

Table I Group differences in cognitive scores: mean (s.d.)

\begin{tabular}{|c|c|c|c|c|c|}
\hline & $\begin{array}{l}\text { Normal control } \\
\text { group } \\
(n=\text { II })\end{array}$ & $\begin{array}{l}\text { APS group } \\
\qquad(n=12)\end{array}$ & $\begin{array}{l}\text { PPS group } \\
\qquad(n=I I)\end{array}$ & $F$ & $P$ \\
\hline RPM, \% & $82.73(14.21)$ & $78.75(16.25)$ & $68.64(19.25)$ & 2.09 & 0.140 \\
\hline WCST: no. of concepts attained & $5.45(1.29)$ & $4.83(1.64)$ & $3.45(1.57)$ & 5.04 & 0.013 \\
\hline WCST: no. of perseverative errors & $2.91 \quad(3.18)$ & $1.83(1.34)$ & $12.27(6.48)$ & 21.20 & 0.000 \\
\hline WCST: total no. of errors & $6.64(8.15)$ & 8.08 (7.38) & $19.54(9.43)$ & 7.88 & 0.002 \\
\hline NART & $112.18(3.15)$ & $104.08(11.67)$ & $102.73(9.39)$ & 3.62 & 0.039 \\
\hline FAS & $36.09(6.28)$ & $23.67(10.33)$ & 24.09 (9.55) & 6.95 & 0.003 \\
\hline SET & $61.45(7.70)$ & $41.58(11.18)$ & $42.00(11.53)$ & 13.54 & 0.000 \\
\hline
\end{tabular}

APS, adequately performing schizophrenia patients; PPS, poorly performing schizophrenia patients; RPM, Raven's Progressive Matrices; WCST, Wisconsin Card Sorting Test; NART, National Adult Reading Test; FAS, controlled oral word association test; SET, SET test (see text for details of tests).
Subjects repeated the WCST as part of the SPECT cognitive activation procedure. Initial (CA1, PE1, TE1) and repeat (CA2, PE2, TE2) test performances were compared. Subjects overall showed no change, but there was a significant group-occasions interaction for preseverative error scores $(F=0.986 ; \quad P<0.000)$, which was due principally to a decrease in the PPS group score on re-testing (mean preseverative error score change for normal control group -0.36 , s.d. $=1.69$; for APS group, -5.45 , s.d. $=5.77$; controls $v$. PPS $t=2.81, \quad P=0.016)$. Consequently group mean perseverative error score differences at the time of SPECT cognitive activation were no longer significant $(F=2.780$; $P=0.078$ ).

\section{Regional CBF during the control condition and following activation}

The $\mathrm{rCBF}$ relative to cerebellar $\mathrm{CBF}$ was measured in 26 ROIs (10 cortical regions on each side, the caudate and the thalamus on each side, and whole brain slices at ventricular and supraventricular levels).

Regional CBF during the control condition varied considerably: the lowest rate for a particular ROI was recorded in the left superior-parietal region (mean $=0.72$, s.d. $=0.05)$, the highest in the right caudate (mean $=0.91$, s.d. $=0.09$ ). The effect of cognitive activation on $\mathrm{rCBF}$ was to produce an overall increase, but with substantial regional variations (Fig. 1). Percentage increase ranged from 0.09 (right caudate) to 7.47 (inferior prefrontal).

Group differences during the control condition and during activation were 2.67, s.d. $=4.62$; and for PPS group analysed. Effects due to age and to antipsychotic drug medication (measured in chlorpromazine equivalents) were explored using analysis of covariance (ANCOVA) and shown to be insignificant. The variance due to these effects was then removed. Group differences did not achieve significance at the $5 \%$ level for any of the 26 ROIs. The two schizophrenia groups were then merged, and a comparison of patient $v$. normal control group undertaken. No significant group differences were detected.

The effect of cognitive activation was then examined by subtracting $\mathrm{rCBF}$ in the control condition from rCBF during activation, and using the values of the change as the dependent variable. In a two-group (schizophrenia $v$. control) comparison, differences were apparent in the left anterior cingulate region $(P=0.043$; schizophrenia group, mean $=0.047$, s.d. $=0.084$; control group, -0.014 , s.d. $=0.071)$ and for whole slice $2(P=0.028$; schizophrenia group, mean $=0.028$, s.d. $=0.036$; control group, -0.005 , s.d. $=0.032$ ). In a threegroup comparison, group differences were only significant at the $10 \%$ level (left anterior cingulate: $P=0.070$; PPS group, mean $=$ 0.061 , s.d. $=0.107$; APS group, 0.035, s.d. $=0.056$; normal control, -0.014 , s.d. $=0.071$ : whole slice 2: $P=0.086$; PPS group, mean $=0.024$, s.d. $=0.046$; APS group, 0.032 , s.d. $=0.026$; normal control, -0.005 , s.d. $=0.032)$. Regional cerebral blood flow values (mean and s.d.) for the three groups during the control condition are shown in Table 2; change values are shown in Table 3.

An alternative analytical approach was also used. The two-group and three-group analyses were repeated, using analysis of covariance in which the $\mathrm{rCBF}$ values during the WCST were used as the dependent variable and $\mathrm{rCBF}$ during the control task as an additional covariate. None of the differences were significant.

\section{Relationship between regional cerebral blood flow activation and cognitive performance}

Pearson correlations were calculated between the rCBF for those ROIs (right inferior frontal, left inferior frontal, left parietal-occipital, left superior frontal, lower whole slice, left superior frontalmiddle segment) that showed the greatest increase in $\mathrm{rCBF}$ during activation and the principal measures of the activation: WCST, the CA2, PE2 and TE2. The left 
Table 2 Mean regional blood flow values (s.d.) acquired during the control task. Values are expressed as ratios to mean cerebellar regional cerebral blood flow

\begin{tabular}{|c|c|c|c|c|c|c|}
\hline & \multicolumn{2}{|c|}{ Normal control group $(n=I I)$} & \multicolumn{2}{|c|}{ APS group $(n=12)$} & \multicolumn{2}{|c|}{ PPS group $(n=I I)$} \\
\hline & Left & Right & Left & Right & Left & Right \\
\hline Frontal pole & $0.885(0.080)$ & $0.915(0.086)$ & $0.855(0.061)$ & $0.878(0.082)$ & $0.847(0.098)$ & $0.861(0.079)$ \\
\hline Inferior prefrontal & $0.799(0.063)$ & $0.787(0.080)$ & $0.789(0.074)$ & $0.757(0.073)$ & $0.763(0.052)$ & $0.752(0.065)$ \\
\hline Superior temporal & $0.825(0.066)$ & $0.855(0.044)$ & $0.806(0.064)$ & $0.814(0.069)$ & $0.772(0.048)$ & $0.810(0.050)$ \\
\hline Inferior parietal-occipital & $0.764(0.060)$ & $0.787(0.056)$ & $0.777(0.053)$ & $0.792(0.048)$ & $0.753(0.054)$ & $0.790(0.049)$ \\
\hline Caudate & $0.906(0.08 \mathrm{I})$ & $0.880(0.081)$ & $0.895(0.104)$ & $0.928(0.093)$ & $0.906(0.064)$ & $0.919(0.081)$ \\
\hline Thalamus & $0.916(0.112)$ & $0.924(0.083)$ & $0.919(0.074)$ & $0.870(0.083)$ & $0.850(0.079)$ & $0.872(0.064)$ \\
\hline Whole slice at ventricular level & \multicolumn{2}{|c|}{$0.734(0.048)$} & \multicolumn{2}{|c|}{$0.724(0.055)$} & \multicolumn{2}{|c|}{$0.712(0.029)$} \\
\hline Superior frontal (anterior) & $0.774(0.069)$ & $0.808(0.07 \mathrm{I})$ & $0.743(0.064)$ & $0.775(0.075)$ & $0.734(0.055)$ & $0.753(0.063)$ \\
\hline Superior frontal (mid) & $0.784(0.063)$ & $0.802(0.068)$ & $0.750(0.082)$ & $0.783(0.059)$ & $0.722(0.069)$ & $0.760(0.055)$ \\
\hline Superior frontal (posterior) & $0.805(0.08 \mathrm{I})$ & $0.788(0.061)$ & $0.758(0.074)$ & $0.760(0.057)$ & $0.757(0.080)$ & $0.754(0.047)$ \\
\hline Superior frontal (combined) & $0.782(0.058)$ & $0.800(0.058)$ & $0.75 I(0.067)$ & $0.774(0.060)$ & $0.739(0.061)$ & $0.757(0.05 I)$ \\
\hline Anterior cingulate & $0.820(0.058)$ & $0.822(0.053)$ & $0.776(0.08 \mathrm{I})$ & $0.785(0.101)$ & $0.762(0.091)$ & $0.772(0.069)$ \\
\hline Superior parietal & $0.729(0.062)$ & $0.74 I(0.046)$ & $0.720(0.053)$ & $0.754(0.072)$ & $0.710(0.054)$ & $0.722(0.037)$ \\
\hline Whole slice at supraventricular level & \multicolumn{2}{|c|}{$0.688(0.037)$} & \multicolumn{2}{|c|}{$0.676(0.051)$} & \multicolumn{2}{|c|}{$0.666(0.039)$} \\
\hline
\end{tabular}

APS, adequately performing schizophrenia patients; PPS, poorly performing schizophrenia patients.

Table 3 Changes in mean cerebral blood flow values (s.d.) with specific activation (regional cerebral blood flow, rCBF) during the Wisconsin Card Sorting Test less rCBF value during control task (values are expressed as ratios to mean cerebellar rCBF)

\begin{tabular}{|c|c|c|c|c|c|c|c|c|c|c|}
\hline \multirow[b]{3}{*}{ Frontal pole } & \multicolumn{3}{|c|}{ Normal control group $(n=I I)$} & \multicolumn{4}{|c|}{ APS group $(n=12)$} & \multicolumn{3}{|c|}{ PPS group $(n=I I)$} \\
\hline & \multicolumn{2}{|c|}{ Left } & \multirow{2}{*}{$\begin{array}{c}\text { Right } \\
0.026(0.172)\end{array}$} & \multicolumn{2}{|c|}{ Left } & \multicolumn{2}{|c|}{ Right } & \multicolumn{2}{|c|}{ Left } & \multirow{2}{*}{$\begin{array}{c}\text { Right } \\
0.019(0.194)\end{array}$} \\
\hline & 0.026 & $(0.213)$ & & 0.042 & $(0.075)$ & -0.009 & $(0.083)$ & 0.031 & $(0.096)$ & \\
\hline Inferior prefrontal & 0.056 & $(0.121)$ & $0.052(0.170)$ & 0.043 & $(0.046)$ & 0.055 & $(0.055)$ & 0.059 & $(0.049)$ & $0.052(0.060)$ \\
\hline Superior temporal & -0.004 & $(0.074)$ & $0.030(0.113)$ & 0.010 & $(0.049)$ & 0.039 & $(0.055)$ & 0.040 & $(0.057)$ & $0.024(0.048)$ \\
\hline Inferior parietal-occipital & 0.052 & $(0.109)$ & $0.041(0.130)$ & 0.022 & $(0.057)$ & 0.053 & $(0.088)$ & 0.037 & $(0.058)$ & $0.031(0.049)$ \\
\hline Caudate & 0.031 & $(0.113)$ & $0.034(0.172)$ & 0.017 & $(0.080)$ & \multicolumn{2}{|c|}{$-0.033(-0.083)$} & 0.003 & $(0.065)$ & $-0.018(0.100)$ \\
\hline Thalamus & 0.053 & $(0.194)$ & $0.060(0.140)$ & -0.004 & $(0.159)$ & 0.064 & $(0.115)$ & 0.003 & $(0.103)$ & $0.012(0.135)$ \\
\hline Whole slice at ventricular level & \multicolumn{3}{|c|}{$0.014(0.092)$} & \multicolumn{4}{|c|}{$0.032(0.032)$} & \multicolumn{3}{|c|}{$0.024(0.04 I)$} \\
\hline Superior frontal (anterior) & -0.003 & $(0.073)$ & $-0.009(0.069)$ & 0.028 & $(0.063)$ & 0.023 & $(0.064)$ & 0.049 & $(0.062)$ & $0.026(0.085)$ \\
\hline Superior frontal (mid) & 0.002 & $(0.053)$ & $-0.002(0.053)$ & 0.045 & $(0.072)$ & 0.024 & $(0.058)$ & 0.088 & $(0.078)$ & $0.042(0.109)$ \\
\hline Superior frontal (posterior) & 0.023 & $(0.086)$ & $-0.004(0.070)$ & 0.047 & $(0.068)$ & 0.042 & $(0.049)$ & 0.011 & $(0.075)$ & $0.035(0.077)$ \\
\hline Superior frontal (combined) & 0.003 & $(0.065)$ & $0.000(0.060)$ & 0.042 & $(0.061)$ & 0.031 & $(0.050)$ & 0.057 & $(0.064)$ & $0.035(0.077)$ \\
\hline Anterior cingulate & $-0.014^{*}$ & $(0.070)$ & $-0.016(0.099)$ & $0.035^{*}$ & $(0.056)$ & 0.017 & $(0.069)$ & $0.061 *$ & $(0.107)$ & $0.048(0.103)$ \\
\hline Superior parietal & 0.010 & $(0.062)$ & $0.003(0.055)$ & 0.023 & $(0.039)$ & 0.026 & $(0.060)$ & 0.025 & $(0.068)$ & $0.026(0.080)$ \\
\hline
\end{tabular}

$* P<0.1$.

APS, adequately performing schizophrenia patients; PPS, poorly performing schizophrenia patients.

anterior cingulate was included among the ROIs because, although it did not show a very significant increase in activation $(4.02 \%)$, it is this region that discriminates best between groups. There were thus 21 calculations. When all subjects were included, none of the correlations achieved significance. When the three groups were each studied separately, a pattern emerged: in the normal control group, $\mathrm{rCBF}$ for all of the ROIs correlated positively with
CA2 scores, and negatively with PE2 and TE2 scores, while in the APS group, the reverse was true in 15 of the 18 calculations. The PPS group resembled the APS group. However, the only significant correlations were in the APS group between the left superior frontal (middle segment) and CA2 (Pearson correlation -0.765 , $P<0.01$ ) and left superior frontal (middle segment) and TE2 (Pearson correlation 0.667, $P<0.05$ ).

\section{DISCUSSION}

The principal aim of the study was to attempt to replicate and extend the finding reported by Weinberger et al (1986). They found that subjects with schizophrenia performed poorly on the WCST (which is thought to depend, at least in part, upon the integrity of frontal lobe function), and failed to exhibit a significant increase in dorsolateral prefrontal cortical rCBF such 
as could be detected in normal subjects while performing the test. This failure could not be readily explained as part of a general inability to respond to cognitive challenge with appropriate regional activation, as the same group of patients did not differ from normal subjects in their rCBF response to RPM, a non-frontal cognitive challenge.

\section{Technical and methodological limitations}

Limitations of technique and methodology must be acknowledged. The radiotracer technique employed provides an estimate of $\mathrm{rCBF}$ relative to a common reference region so that different regions may be compared: it does not measure $\mathrm{rCBF}$ as such. SPECT lacks the spatial resolution of positron emission tomography (PET) and functional magnetic resonance imaging (fMRI). The cerebellum was chosen as the reference ROI. It is an easily identifiable and definable ROI, and, although it may be implicated in the schizophrenic process (see, for example, Volkow et al, 1992) the evidence is less compelling than for the cerebral hemispheres, the neocortices of which are almost certainly extensively involved. Structural image coregistration would have reduced movement artefact and improved anatomical interpretation, but was not available to us. Nor did we have access to a SPECT programme that provided a three-dimensional reconstruction. Although the performance of the normal control group was superior to that of the patient group on a measure of premorbid intelligence, the groups did not differ on the RPM, a current measure of general intelligence, and it seems unlikely that this discrepancy would have significantly influenced the outcome. Finally, the analysis involved multiple comparisons, but the variables - regional increases in blood flow - were highly correlated and a Bonferroni correction would have been excessively conservative.

Our study was designed to investigate not only those patients who performed poorly on the WCST, but also those whose performance did not differ significantly from that of normal controls. We were able to identify groups of poorly performing (PPS) and adequately performing (APS) patients with schizophrenia. All groups performed adequately on the RPM. Measurement of rCBF during the control task did not show any between-group differences. Performance on the sorting task was associated with widespread cortical activation, as judged by increase in $\mathrm{rCBF}$ (especially in the frontal and occipital egions). Test performance related to $\mathrm{rCBF}$ increase did not differ greatly between groups, but a direct comparison of the combined group of patients with the normal control group showed the former to have a greater increase in the left cingulate region and in the whole transverse slice at supraventricular level. In the normal control group we detected a tendency for the size of the rCBF performance-related increase to be directly related to the test performance scores, while in the groups of patients the reverse was the case. The PPS group showed a highly significant improvement in perseverative error scores between the two test occasions.

\section{Differences in cohort characteristics may explain the failure to replicate}

We were unable to replicate Weinberger $e t$ al's (1986) findings. Many of our patients were able to obtain respectable scores on the WCST, and many of those who failed to do so the first time showed a marked practice effect. Patients and controls alike showed a fronto-occipital performancerelated increase in blood flow (in some regions this was marginally greater for patients with schizophrenia than for controls).

Why should our results differ? A clue may lie in the failure of most of Weinberger et al's patients to perform the WCST or to benefit from intense guidance and coaching (Goldberg et al, 1987). This raises the possibility that they were considerably more impaired, which is further supported by the clinical description provided. These studies drew upon a cohort of patients who had been selectively referred to a national clinical research centre and were refractory to conventional treatment. The subjects had been withdrawn from antipsychotic medication 4 weeks before participating in the study, and, although this interval would be insufficient to ensure tissue clearance, it is likely that in many cases clinical deterioration would compromise test performance. Our patients were stable, medicated out-patient attenders, drawn from local district services, to which they had been referred by general practitioners. The services in the two hospitals were provided for a provincial and for an inner-city population, respectively; they may therefore be regarded as a fairly representative sample of patients with schizophrenia.

There have been few other published attempts to replicate these results. Kawasaki et al (1993) used a similar neuroimaging technique to that described here, but used as a control condition the subject in a resting state in the imaging position. Both patient and normal control groups performed adequately on the WCST, and both showed a performance-related $\mathrm{rCBF}$ increase in the left dorsolateral prefrontal cortex. Cantor-Graae et al (1991), in an uncontrolled study of seven patients with chronic schizophrenia, using the xenon inhalation method, failed to demonstrate any regional activation. However, the same group (Cantor-Graae et al, 1993) failed to show activation in normal subjects. More recent studies using fMRI have demonstrated prefrontal cortical activation with WCST.

\section{Use of the WCSTas a means of prefrontal cortex activation}

Our failure to replicate Weinberger et al's findings may be due to patient selection; other factors may also play a part. Activation of a discrete area of the dominant hemisphere dorsolateral prefrontal cortex (Brodmann's area 46) during performance of the WCST in normal subjects is a consistent finding of Weinberger's group (Weinberger et al, 1986, 1988 ) and is central to their hypothesis.

The value of WCST as a means of localising brain dysfunction was first demonstrated in subjects with discrete frontal lobe lesions. This may not be an appropriate model for schizophrenia. Even within samples of neurological patients, the test has failed to distinguish persons with frontal lesions from those with more diffuse brain disease (Robinson et al, 1980).

However, in our study frontal activation did occur, but was widespread throughout the frontal regions in both hemispheres. This is hardly surprising. Even the simplest cognitive tasks are mediated through a parallel distributed network, as demonstrated by a multifocal activation pattern. The WCST is a complex task that makes demands on a number of resources, including, among others, focused attention, working memory and conceptual flexibility. Activation limited to a discrete cortical area is not to be expected. Occipital 
activation was also seen and has previously been reported during the processing of complex visual stimuli (Phelps et al, 1981).

Finally, the widely accepted view, based on early studies, that performance on the WCST is specifically impaired in schizophrenia is increasingly open to question. In a study of chronic stable medicated out-patients comparable to our own (Braff, 1991), subjects completed a comprehensive battery of 20 neuropsychological measures. The WCST ranked only 17th in terms of its sensitivity to schizophrenic deficit; however, one-third of the patients fell in the impaired range on perseverative response scores; poor performance was associated with the presence of negative symptoms. On a battery of standardised tests, untreated schizophrenia sufferers showed a global deficit, but were less impaired on the WCST than on most other tests (Saykin et al, 1991). Other studies continue to report impaired WCST performance (Stratta et al, 1993).

This lack of agreement may reflect clinical heterogeneity. It may also be due to procedural differences, in particular the use of both the standardised and the modified forms of the WCST and the increasing dependence on automated presentation.

\section{Stability of WCST over time}

Goldberg et al (1987) reported that their subjects with schizophrenia did not improve their performance on the WCST, even after they were given additional instructions while taking the tests. All of our subjects repeated the test, often after an interval of several months. On each occasion they received the same set of instructions. No attempts were made to discuss with them the principles underlying the test, or how their performance might be improved. Nevertheless, the test scores of the PPS group improved considerably, and although their performance was still inferior to that of the other groups at the time of neuroimaging, this was no longer significant. Part of the improvement could be explained as a regression towards the mean, but this would assume that the initial scores were randomly distributed and inherently lacking in constancy, whereas the strong correlations with intelligence, educational attainment, and social and occupational achievement established elsewhere suggest that performance on the WCST is a fairly stable characteristic. Our

\section{CLINICAL IMPLICATIONS}

- The study was unable to confirm a failure of prefrontal activation in schizophrenia as previously reported.

- The performance of a test of executive function (the Wisconsin Card Sorting Test ) was accompanied by a widespread increase in frontal blood flow. Subjects with schizophrenia showed this increase regardless of whether they performed adequately or poorly on the test.

Poor performance on tests of executive function is not irremediable. This has important implications for rehabilitation.

\section{LIMITATIONS}

- Image co-registration would have reduced movement artefact and improved anatomical identification.

- The use of parametric statistical mapping would have increased accuracy and precision.

- The groups could have been more closely matched for age.

B. K. TOONE, FRCPsych, C. I. OKOCHA, MRCPsych, K. SIVAKUMAR, FRCPsych, G. M. SYED, PhD, Departments of Psychological Medicine and Nuclear Medicine, King's College Hospital, London

Correspondence: B. K. Toone, Department of Psychological Medicine, King's College Hospital, Denmark Hill, London SE5 9RS. Tel/Fax: 02073463226

(First received 18 February 1998, final revision 17 March 2000, accepted 17 March 2000)

findings could be due to a practice effect, and as such would be in keeping with other reports of WCST improvement after practice (for example, Metz et al, 1994), and suggest that whatever deficiencies underlie poor performance, they are not irremediable. This much is recognised by Goldberg and Weinberger, who in a recent review (1994) acknowledged that instruction (Green et al, 1990) and even monetary reward (Summerfelt et al, 1990) might lead to improved performance, but questioned whether it would be sustained and the extent to which it might generalise.

We also wished to determine whether the groups of schizophrenia sufferers exhibited different patterns of regional activation from the control groups. Group differences were only evident in the left anterior cingulate region and in the superior transverse plane. The possible significance of the first findings may be somewhat compromised by the observation that this region showed rather less activation than other dominant hemisphere frontal lobe regions. Moreover, in a subsequent analysis of covariance in which the activation $\mathrm{rCBF}$ formed the dependent variable and the control condition $\mathrm{rCBF}$ was used as a covariate, significance was lost.

\section{REFERENCES}

American Psychiatric Association (1987) Diagnostic and Statistical Manual of Mental Disorders (3rd edn, revised) (DSM-III-R). Washington, DC: APA.

Annett, M. (1970) The classification of hand preference by association analysis. British Journal of Psychology, $\mathbf{6}$, 278-296.

Berg, E.A. (1948) A simple objective test for measuring flexibility in thinking. Journal of General Psychology, 39, 15-22.

Braff, D. L., Heaton, R., Kuck, J., et al (199I) The generalised pattern of neuropsychological deficits in outpatients with chronic schizophrenia with heterogenous Wisconsin Card Sorting Test results. Archives of General Psychiatry, 48, 891-898.

Cantor-Graae, E., Warkentin, S., Franzen, G., et a (199I) Aspects of stability of regional cerebral blood flow in chronic schizophrenia: an 18 year follow-up study. Psychiatry Research, 40, 253-266. 
$\ldots, \ldots$, , et al (1993) Frontal lobe challenge: a comparison of activation procedures during $\mathrm{rCBF}$ measurements in normal subjects. Neuropsychiatry, Neuropsychology \& Behavioural Neurology, 6, 88-92.

\section{Goldberg, D. E. \& Weinberger, D. R. (1994)}

Schizophrenia, training paradigms and the Wisconsin Card Sorting Test redux. Schizophrenia Research, II, 291-296.

_, _, Berman, K. F., et al (1987) Further evidence for dementia of the prefrontal type? Archives of General Psychiatry, 44, 1008-1014.

Green, M. F., Ganzel, S., Satz, B., et al (1990) Teaching the Wisconsin Card Sorting Test to schizophrenic patients (letter). Archives of General Psychiatry, 47, 91-92.

Holman, B. L. (1986) Perfusion and receptor SPECT in the dementias. Journal of Nuclear Medicine, 27, 855-860.

lager, A. C., Kirch, D. G., Wyatt, R. J., et al (1995) A negative symptom rating scale. Psychiatry Research, 16, 27-36.

Isaacs, B. \& Akhtar, A. J. (1972) The SET test: a rapid test of mental function in old people. Age \& Ageing, I, 222-226.

Kawasaki, Y., Maieda, Y., Suzuki, N., et al (1993) SPECT analysis of regional cerebral blood flow changes in patients with schizophrenia during the Wisconsin Card Sorting Test. Schizophrenia Research, 10, 109-116.

Krawiecka, M., Goldberg, D. \& Vaughan, M. (1977) A standardized psychiatric assessment scale for rating chronic psychotic patients. Acta Psychiatrica Scandinavica, 55, 299-308.

Mayfield, D., McLead, G. \& Hall, P. (1974) The CAGE questionnaire validation of a new alcoholism screening instrument. American Journal of Psychiatry, I3I, |I2I-II23.

Metz, J.T., Johnson, M. D., Pliskin, M. H., et al (1994) Maintenance of training effects on the Wisconsin Card Sorting Test by patients with schizophrenia or affective disorders. American Journal of Psychiatry, I5I, 120-122.

Montaldi, D., Brooks, D. \& McColl, J. (1990)

Measurements of cerebral blood flow and cognitive performance in Alzheimer's disease. Journal of Neurology, Neurosurgery \& Psychiatry, 53, 33-38.

Nelson, H. E. (1976) A modified card sorting test sensitive to frontal lobe defects. Cortex, 12, 313-324.

- (1982) National Adult Reading Test (NART). Windsor: NFER-Nelson.

Phelps, M. E., Mazziotta, J. C., Kuhl, D. E., et a (1981) Tomographic mapping of human cerebral metabolism: visual stimulation and deprivation. Neurology, 3I, 517-529.

Raven, J. C. (1988) Raven's Progressive Matrices and Vocabulary Scales. Oxford: Oxford University Press.

Robinson, A. L., Heaton, R. K., Lehman, R. A., et al (1980) The utility of the Wisconsin Card Sorting Test in detecting and localising frontal lobe lesions. Journal of Consulting \& Clinical Psychology, 48, 605-614.

Saykin, A. J., Gur, R. C., Gur, R. E., et al (1991) Neuropsychological function in schizophrenia: selective impairment in memory and learning. Archives of General Psychiatry, 48, 618-624.

Schonell, F. (1942) Backwardness in the Basic Subject. London: Oliver and Boyd.

Sharp, F. P., Smith, F. E., Gemmel, H. P., et al (1986) Technetium-99mHMPAO stereo isomers as potential agents for imaging regional cerebral blood flow: human volunteer studies. Journal of Nuclear Medicine, 27 $17 \mid-177$

Spreen, O. \& Benton, A. L. (1969) Neurosensory Centre Comprehension Examination for Aphasia. Victoria, BC: Neuropsychology Laboratory, University of Victoria.

Stratta, P., Rossi, A., Mancini, F., et al (1993)

Wisconsin Card Sorting Test performance and educational level in schizophrenic and control samples. Neuropsychiatry, Neuropsychology \& Behavioural Neurology, 6, 149-153.

Summerfelt, A. T., Alphs, L. D., Whiteman, A. M. I., et al (1991) Reduction of perseverative errors in patients with schizophrenia using monetary feedback. Journal of Abnormal Psychology, 100, 613-616,

Syed, G. M. S., Eagger, S., Toone, B. K., et al (1992) Quantification of regional cerebral blood flow ( $\mathrm{rCBF}$ ) using ${ }^{99} \mathrm{Tc}^{\mathrm{m}}-\mathrm{HMPAO}$ and SPECT: choice of reference region. Nuclear Medicine Communications, 13, 811-816.

Volkow, N. D., Levy, A., Brodie, J. D., et al (1992) Low cerebellar metabolism in medicated patients with chronic schizophrenia. American Journal of Psychiatry, 149, 686-688.

Weinberger, D. R., Berman, K. F. \& Zec, R. F. (1986) Physiology dysfunction of dorsolateral prefrontal cortex in schizophrenia. I. Regional cerebral blood flow evidence. Archives of General Psychiatry, 43, 114-124.

— \& Illowsky, B. P. (1988) Physiological dysfunction of dorsolateral prefrontal cortex in schizophrenia. III. A new cohort and evidence for a monoaminergic mechanism. Archives of General Psychiatry, 45, 609-615. 